\title{
Fibre Studies
}

$\mathrm{E}^{\mathrm{x}}$ XCEPT in so far as Section A may regard all other sections of the British Association as subsidiary to the mathematical and physical sciences, the subject of fibre structure is by no means a prerogative of physicists sitting alone, and discussion to be most profitable is best conducted with the co-operation of one or more of the other sciences, such as chemistry and biology. This point was emphasized by W. T. Astbury in opening a brief symposium on fibre studies arranged for Section A at the Blackpool meeting of the Association, but his subsequent remarks, dealing with problems arising out of the long-range elasticity of many protein fibres, were addressed specially to physicists.

Certain natural protein fibres, in particular the hairs of mammals, are endowed with elastic properties of relatively enormous range. The effect is shown by X-ray and related studies to be due to combinations of polypeptide chain-molecules, normally in a regularly-folded configuration, from which they may be pulled out straight by the application of tension, and to which they return when the tension is released. By suitable modification of the state of cross-linkage of these polypeptide chains, both the elastic range and the driving force of elastic recovery may be varied considerably. Animal hairs, for example, may actually be contracted to a length only about half their natural length. Closely analogous, from both the X-ray and physical elastic point of view, to one of the modifications of the hair protein, keratin, is the chief muscle protein, myosin, and there is apparently some fundamental type of molecular fold common to both. The crystalline proteins, which are built from 'globular' molecules, have now also been shown to be based most probably on regularly-folded polypeptide chains. The latter are liberated from their special, and often highly specific, configuration by the change known as 'denaturation', the resulting insoluble mass being often highly elastic like keratin and myosin. $\mathrm{X}$-rays suggest that this elasticity is again to be referred to groups of folded polypeptides which may sometimes be drawn out into straight chains, just as keratin and myosin may be so drawn out. By this means artificial protein fibres may be prepared from originally globular molecules.

The detailed interpretation of the deformation and unfolding of protein chain systems provides one of the most fascinating and difficult problems in molecular mechanics. At the moment, it seems to present two chief aspects : (1) Is the hexagonal fold postulated for keratin and myosin sound ? (2) Is the keratin-myosin fold-whatever it may be $-a$ fundamental fold for proteins in general ?

Dr. J. B. Speakman, accepting the X-ray picture and intramolecular stress theory of keratin structure as substantially correct, proceeded to show how the many elastic modifications that arise when strained fibres are treated with various reagents can be simply explained in terms of the reactivity of the salt and disulphide cross-linkages of the keratin 'grid'. The reactivity of these linkages depends on their state of stress, which in turn depends on the configuration of the grid. In consequence, the properties of each type of linkage influence those of the remainder: thus the reactivity of the disulphide linkage in stretched hairs is frequently at a maximum under conditions in which the salt linkages are most stable.

The concept was illustrated by the results of numerous experiments in which the stretched fibres were submitted to the action at $50^{\circ} \mathrm{C}$. of a series of mixtures of sodium sulphite and bisulphite, so chosen as to vary the $p \mathrm{H}$ while maintaining the reducing power. The extent of disulphide breakdown was then estimated by measuring the amount of 'permanent set' produced. It is a

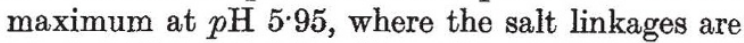
most stable, and falls off rapidly on either side of this point as their stability decreases. The interpretation put forward by Speakman is that the dissolution of the salt linkages permits a configurational rearrangement which relieves the stress on the disulphide linkages and so reduces their reactivity. In the hands of Speakman, this chemical modification and 'playing off' of one linkage against another has proved exceedingly fruitful, and the properties of hairs so modified present features of unusual interest, whether for chemist or physicist.

The rather more pragmatic side of fibre research was emphasized by the contributions of Dr. M. Mathieu on "The X-Ray Cinematography of a Simple Fibre Reaction", and of Dr. E. Griffiths and J. H. Awbery on "Apparatus for Maintaining Constant Humidity". Mathieu introduced a new example of powerful X-ray tube with rotating anticathode, the latter being shut off from the atmosphere by nothing more elaborate than a ground cone, suitably lubricated with vacuum grease and under the control of an external adjustable spring to prevent 'seizing'. The rate of rotation possible with this system is not high-one or two revolutions per second-but it has sufficed to take one-minute 
photographs of the nitration of cellulose with gaseous nitrogen pentoxide, and so to follow the process step by step. The regularity of the original cellulose structure first disappears in the direction of the fibre-axis, until a stage is reached where there remain only equatorial reflections and the pseudoperiod corresponding to the length of a glucose residue. The molecular chains then move farther apart and slide over one another so as to leave the effective residue thickness unaltered, whereupon the (101) reflection of nitro-cellulose appears. After this, little by little, the chains take up their final stable configuration and the characteristic fibreperiod $(25 \cdot 1 \mathrm{~A}$.) of trinitro-cellulose can be observed.

The whole experiment takes only an hour, and illustrates most strikingly the immediate advantages to be derived from the use of powerful $\mathrm{X}$-ray tubes. Indeed, such tubes are rapidly becoming an absolute necessity. The study of unstable systems and of physical and chemical transformations, particularly those associated with life processes, still offers an enormous field to the structure analyst. We need X-ray cinematograph films of all these things.

W. T. A.

\section{Food Investigation: a Retrospect and the Future}

$\mathrm{T}$ HE reconstitution of the Food Investigation Board in the autumn of 1934 provided an occasion for a review of the work accomplished by the Board during the seventeen years of its existence and the determination of the lines along which further progress should be sought.* Not only have general solutions been found, in the gasstorage of fruit and chilled meat and in the brinefreezing of fish, for the three main problems with which the Board was faced when originally established, but at the same time great advances have also been made in the science that underlies the storage and handling of foodstuffs.

Although each animal product presents its own problems of handling and storage, yet, in the final analysis, these problems may be stated more generally as those of the properties and behaviour of proteins and fats, of pigments and micro-organisms. Thus, among the main subjects of investigation have been rigor mortis and glycogenolysis in muscle; the identity and physical chemistry of animal proteins ; the freezing of colloidal systems and tissues ; the chemistry of the pigments ; the constitution of the animal fats ; and the identification and characterization of the micro-organisms found on animal products. The Board now considers that it would be profitable to take up other lines of work as opportunity offers, the most important being the organic chemistry of the proteins. It is now known, for example, that the protein myosin controls the changes taking place in meat and fish during rigor mortis, as well as the structural changes that occur during storage : in the egg, the protein mucin is the important factor. Progress has been made in determining the general physico-chemical properties of the proteins, but further advances will depend upon

* Department of Scientific and Industrial Research. Report of the Food Investigation Board for the Year 1935. Pp. $x+232$. (London: H.M. Stationery Office, 1936.) 38. 6d. net. an exact knowledge of the constituent amino acids and of their arrangement within the molecule. The results of such investigations will be of wide interest in chemistry, physiology and nutrition. The Board has therefore arranged for a member of the director's staff to spend a year in Prof. A. C. Chibnall's laboratory at the Imperial College of Science and Technology in order to gain experience of the specialized technique required, before under. taking research on this subject. It is also hoped to commence work on the identification of the autolytic enzymes of meat and eggs and the lipolytic enzymes of bacteria, to the action of which is often due the development of taint in animal foodstuffs. Although attention has hitherto been devoted chiefly to meat, the programme of research has now been widened to include both eggs and poultry, and the problems of their wastage and quality.

The handling and storage of fresh fruit present problems different in character from those of animal produce, since the material is living and not dead, and because the plants concerned belong to different orders and genera, and the parts used as foodstuffs comprise nearly all their chief organs. In spite of the work which has been carried out on the preservation of fruit and vegetables, the qualities after such periods of storage as are customary in commerce are, in general, inferior to those of the material at its best. The extension of control depends upon the advancement of our knowledge of the physico-chemical mechanism upon which the living processes are based. Up to the present, the Board has concentrated upon an intensive study of the respiration and metabolism of carbohydrate in the apple and potato. It is now including a study of the enzymes and the metabolism of nitrogen, and intends to investigate the metabolism of acid, the formation and 